\title{
Impact of mammalian cell culture conditions on monoclonal antibody charge heterogeneity: an accessory monitoring tool for process development
}

\author{
Bernhard Sissolak $^{1}$ D $\cdot$ Nico Lingg $^{2} \cdot$ Wolfgang Sommeregger $^{3} \cdot$ Gerald Striedner $^{1} \cdot$ Karola Vorauer-UhI $^{1}$
}

Received: 31 October 2018 / Accepted: 30 May 2019 / Published online: 7 June 2019

(c) The Author(s) 2019

\begin{abstract}
Recombinant monoclonal antibodies are predominantly produced in mammalian cell culture bioprocesses. Post-translational modifications affect the micro-heterogeneity of the product and thereby influence important quality attributes, such as stability, solubility, pharmacodynamics and pharmacokinetics. The analysis of the surface charge distribution of monoclonal antibodies provides aggregated information about these modifications. In this work, we established a direct injection $\mathrm{pH}$ gradient cation exchange chromatography method, which determines charge heterogeneity from cell culture supernatant without any purification steps. This tool was further applied to monitor processes that were performed under certain process conditions. Concretely, we were able to provide insights into charge variant formation during a fed-batch process of a Chinese hamster ovary cell culture, in turn producing a monoclonal antibody under varying temperatures and glucose feed strategies. Glucose concentration impacted the total emergence of acidic variants, whereas the variation of basic species was mainly dependent on process temperature. The formation rates of acidic species were described with a second-order reaction, where a temperature increase favored the conversion. This platform method will aid as a sophisticated optimization tool for mammalian cell culture processes. It provides a quality fingerprint for the produced $\mathrm{mAb}$, which can be tested, compared to the desired target and confirmed early in the process chain.
\end{abstract}

Keywords Product quality $\cdot$ Recombinant mAbs $\cdot$ Charge heterogeneity determination $\cdot$ Mammalian cell culture $\cdot \mathrm{CHO}$

\section{Introduction}

Recombinantly produced monoclonal antibodies (mAbs), as well as biosimilars, are key products in today's pharmaceutical industry [1, 2]. Post-translational product modifications induced by chemical and enzymatical intra- and extracellular mechanisms during the production process lead to micro-heterogeneity of mAbs, in turn affecting product characteristics (e.g., efficacy, safety, pharmacodynamics and pharmacokinetics) [3]. The recombinant cell line, the culture media and the process settings affect these quality

Bernhard Sissolak

bernhard.sissolak@boku.ac.at

1 Department of Biotechnology, University of Natural Resources and Life Sciences (BOKU), Vienna, Austria

2 Austrian Centre of Industrial Biotechnology GmbH (ACIB), Vienna, Austria

3 Bilfinger Industrietechnik Salzburg GmbH, Salzburg, Austria attributes $[4,5]$. During process development, it is important to ensure a reproducible, distinct and preferably homogenous pattern of the product. For the establishment of biosimilars, it is important to match the characteristics of the originator product [6]. The effects of various extra- and intracellular influences on different aspects of product quality have been evaluated in great detail. For instance, $N$-glycosylation is by far the best-studied quality attribute and there are several strategies available for glycosylation control [7].

One additional important measure of $\mathrm{mAb}$ heterogeneity is the distribution of surface charge variants. Due to numerous modifications, the net surface charge of mAbs can be altered [8-11]. Charge species with a lower isoelectric point (pI) than the main fraction of the product are defined as acidic variants and generated by sialylation, deamidation of asparagine and glutamine, glycation and other mechanisms. Glycation, for instance, is a non-enzymatic reaction where a reducing sugar molecule, most commonly glucose, is covalently bound to a reactive amino group [12]. Basic variants are defined as species with a higher $\mathrm{pI}$ than the main fraction 
and generated by incomplete C-terminal lysine clipping of the heavy chains, as well as by fragmentation and aggregation [13]. Several studies indicate that $\mathrm{mAb}$ variants can lead to varying biological responses [14-16]. For instance, it was shown that the basic variants exhibited an increased binding to the FC and the neonatal receptor, indicating an increased half-life [15]. Another study reported that only a few specific variants of the tested $\mathrm{mAb}$ had a statistical relevant impact on the cell proliferation assay [10]. Hence, to know and understand the mechanism behind the charge heterogeneity is of particular importance.

Common analytical methods for the determination of charge heterogeneities of mAbs are capillary isoelectric focusing (cIEF) and ion exchange chromatography (IEX) [17]. Both methods are widely used in various applications [18], but IEX methods, using a salt gradient elution, are recognized as the gold standard and routinely in use [19-22]. The major limitation of IEX is when using a salt buffer system to coerce the user to adapt it for every new kind of $\mathrm{mAb}$. However, the use of $\mathrm{pH}$ gradients was shown to be product-independent [23] and recently, a cation exchange chromatography (CEX) method with a linear $\mathrm{pH}$ gradient for the determination of charge heterogeneity of mAbs was published [24]. This technique was shown to be robust, exhibit a high resolution [25], result in similar precisions compared to imaged cIEF [26] and be scalable for semi-preparative purposes $[14,15]$.

Monitoring and controlling of product quality are required for the whole production chain [27]. The successful application of process analytical technology (PAT) and quality by control $(\mathrm{QbC})$ to bioprocesses [28] requires reliable and unbiased product quality data over the time course of a fermentation process. Samples taken from crude culture supernatants should be analyzable with a minimum of manipulation. In this respect, pre-purification of relevant samples would possibly falsify the results [29-31]. Moreover, the avoidance of purification steps reduces the workload, while the method becomes more applicable as a processmonitoring tool and allows for decision making early in the process chain.

In this work, we aimed to adjust the method developed by Lingg et al. [24] for the measurement of charge heterogeneity directly from cell culture supernatants without prior purification or additional sample manipulation. This approach offers the possibility to assess any quality changes already in the early stages of cell line, media and process development. Eventually, the derived data enable advanced process characterization and monitoring. In the first part of this manuscript, we explain the applicability of CEX separation for the analysis of crude culture supernatants and evaluate the influence of matrix effects. In the following, it is used as a process-monitoring tool for a model antibody, expressed in Chinese hamster ovary $(\mathrm{CHO})$ cells, within an experimental setting, while varying glucose concentration in the feed media and cultivation temperature. Process relevant samples were analyzed by CEX to study the impact of these variations on $\mathrm{mAb}$ charge heterogeneity.

\section{Materials and methods}

\section{Fed-batch experiments}

A recombinant $\mathrm{CHO}$ monoclonal cell line, generated by the Rosa26 bacterial artificial chromosome expression strategy [32], producing an antitumor necrosis factor (TNF) alpha IgG1, was used (Antibody Lab GmbH, Austria). The cell line originated from the host cell CHO-K1 (ATCC CCL61), which was serum-free adapted for prior use. A working cell bank of the recombinant cell line with $5 \times 10^{6}$ cells per vial was used as the starting material for all experiments. The cells were thawed in chemically defined culture medium (Dynamis AGT, A26175, Thermo Fisher Scientific, USA) supplemented with $8 \mathrm{mM}$ L-glutamine (25030081, Sigma Aldrich, Germany), $3 \mathrm{~mL} / \mathrm{L}$ phenol red solution (RNBD642, Sigma Aldrich, Germany), 1:1000 anti-clumping agent (0010057DG, Thermo Fisher Scientific, USA) and $1 \mathrm{mg} /$ mL G418 (10131027, Thermo Fisher Scientific, USA).

The culture was subsequently passaged three times (every 3-4 days) in the above-mentioned media without G418 and anti-clumping agent and used as the starting material for the inoculation of the batch with a starting cell density of $2.5 \times 10^{5}$ cells $/ \mathrm{mL}$. The fed-batch cultivations were performed in shake flask (\#431147, Corning, USA) with a starting volume of $300 \mathrm{~mL}$. As batch medium, the culture medium was additionally supplemented with $0.1 \%$ (v/v) Antifoam C (A8011, Sigma Aldrich, Germany) to represent typical large-scale cultivation conditions. Within the experimental setup, the parameters of temperature and glucose addition during the feed phase were changed. In this study, the feed (CHO CD EfficientFeed ${ }^{\mathrm{TM}}$ A AGT ${ }^{\mathrm{TM}} \mathrm{Kit}$, A1442002, Thermo Fischer Scientific, USA) was supplemented with $0.1 \%$ antifoam as well as additional 10,20 or $30 \mathrm{~g} / \mathrm{L}$ glucose, which will be referred to as Feed 1, Feed 2 and Feed 3, respectively. The pulse feeding started at day 3 and lasted until day 13. A linear feed rate was carried out with a total added feed volume of $33 \mathrm{vol} \%$ (v/v) with respect to the end volume. The process temperatures were changed at day $4-31{ }^{\circ} \mathrm{C}$ or $34{ }^{\circ} \mathrm{C}$ or remained constant at $37{ }^{\circ} \mathrm{C}$.

An $11 \mathrm{~mL}$ sample was drawn each day for several offline analyses. The cultivations were terminated when the viability dropped below a threshold of $70 \%$. All cultivations were conducted in a humidified $\mathrm{CO}_{2}$ incubator (Heracell ${ }^{\mathrm{TM}}$ VIOs 160i, Thermo Scientific, USA) at $5 \%(\mathrm{v} / \mathrm{v}) \mathrm{CO}_{2}$ in ambient air, at the temperature defined in the experimental design 
with an orbital shaker (MaxQ $2000 \mathrm{CO}_{2}$ Plus, Thermo Scientific, USA) at $200 \mathrm{rpm}$.

For the mock control fed-batch bioprocess, the host cell line was cultivated at a constant $37^{\circ} \mathrm{C}$ with Feed $3(+30 \mathrm{~g} / \mathrm{L}$ glucose). The fed-batch was performed applying the same settings as mentioned above.

\section{All experiments were carried out in duplicates}

For the LC-MS and the boronate affinity chromatography analysis, samples from a stirred tank reactor $(V=15 \mathrm{~L})$ were used. The same procedure, cell line and parameters as stated above were utilized.

\section{Analytics}

The total cell concentration (TCC) was determined by counting the cell nuclei using the particle counter Z2 (Beckman Coulter, USA). Therefore, an aliquot of the cell suspension was centrifuged for $10 \mathrm{~min}$ at $200 \mathrm{~g}$ at room temperature. The cell pellet was resuspended in a $0.1 \mathrm{M}$ citric acid monohydrate (C1909, Merck, Germany) and 2\% (v/v) Trition X-100 (Merck, Germany) buffer. A minimum of $1 \mathrm{~h}$ later, an aliquot of the lysate was diluted with $9 \mathrm{~mL}$ of a $0.9 \% \mathrm{NaCl}$ solution and measured.

Viability was measured by the trypan blue (K490, Amresco, USA) exclusion assay [33]. The viable cell concentration (VCC) was calculated by applying the viability to the TCC.

The product titer was determined by bio-layer interferometry (BLI) using Protein A tips (Octet System, QK, ForteBio, USA) as already described by [34].

The carbohydrates were measured via ion exclusion chromatography (HPX 87H, 300×7.8 mm, \#1250140, BioRad, USA) on an Agilent 1200 series device (Agilent, USA). The column was tempered at $25^{\circ} \mathrm{C}$. The mobile phase was $5 \mathrm{mM}$ sulfuric acid and the flow rate was $0.45 \mathrm{~mL} / \mathrm{min}$. The used detector was a refractive index detector tempered at $35^{\circ} \mathrm{C}$. The calibration range for $\mathrm{D}(+)$-glucose was between 100 and $2000 \mathrm{mg} / \mathrm{L}$. The chromatograms were evaluated with ChemStation software (Revision B.04.01, Agilent, USA).

Gel electrophoresis was performed with an Invitrogen NuPage $^{\text {TM }} 4-12 \%$ Bis-tris gel (NP0321BOX) in a Novex Mini-cell chamber (both Thermo Fisher Scientific, USA). A SeeBlue ${ }^{\circledR}$ Plus2 pre-stained protein standard (LC5925,
Thermo Fisher Scientific, USA) was used for size comparison. The samples were applied with $4 \times$ sample buffer (NuPAGE LDS, NP0007, Thermo Fisher Scientific, USA), while the used running buffer contained $0.3 \%(\mathrm{w} / \mathrm{v})$ Tris, $1.5 \%(\mathrm{w} / \mathrm{v})$ glycine and $0.1 \%(\mathrm{w} / \mathrm{v})$ SDS. Gels were run at 150-200 V. Adalimumab (Humira ${ }^{\mathrm{TM}}$, AbbVie, USA) was used as a reference.

Protein A purification was done via a Proteus Protein A mini spin column (PUR 006, Bio-Rad, USA) according to the manual instructions.

Glycated $\mathrm{mAb}$ species were determined via boronate affinity chromatography (BAC) (0013066, Tosoh Bioscience, Japan). Solvent A consisted of 50 mM EPPS (E9502, Merck, Germany), $10 \mathrm{mM}$ Tris (65837, Fluka, USA) and $200 \mathrm{mM} \mathrm{NaCl}$ (S7653, Merck, Germany), which were adjusted to a pH 8.7 with $10 \mathrm{M} \mathrm{NaOH}$. Solvent $\mathrm{B}$ was $500 \mathrm{mM}$ sorbitol (85529, Merck, Germany) in Mobile Phase A. Chromatography was performed according to a previously published study [35].

For the peptide analysis, the samples were digested in gel and analyzed via LC-MS as previously published [36-38].

\section{Charge heterogeneity determination}

For the method, as previously described in two publications $[24,25]$, a weak cation exchange resin (Dionex ProPac WCX-10 4×250 mm, 088768, Thermo Fisher Scientific, USA) was utilized. Due to the fact that supernatants were directly applied, a guard column $(4 \times 50 \mathrm{~mm})$ was also installed (054994, Thermo Fisher Scientific, USA). Two complex, four-component buffers were used to ensure a highly linear $\mathrm{pH}$ gradient. The compounds were 3-morpholino-2-hydroxypropanesulfonic acid (MOPSO, M8389), 4-(2-hydroxyethyl)piperazine-1-ethanesulfonic acid (HEPES, H3375)), N,N-bis(2-hydroxyethyl)glycine (Bicine, B3879,), 3(cyclohexylamino)-2-hydroxy-1-propanesulfonic acid (CAPSO, C2278) and 3-(cyclohexylamino)-1-propanesulfonic acid (CAPS, C2632, all Merck, Germany). To ensure elution was only based on the $\mathrm{pH}$ shift, sodium chloride (S7653, Merck, Germany) was added to Buffer A according to Table 1 to obtain constant conductivity. The $\mathrm{pH}$ was adjusted with sodium hydroxide (Merck, Germany). The two different buffer systems used are listed in Table 1.

In this study, supernatants were applied using a flow rate of $1.0 \mathrm{~mL} / \mathrm{min}$, while the injection volume was $100 \mu \mathrm{L}$. The
Table 1 Running (A) and elution (B) buffer compositions used in the CEX method

\begin{tabular}{lllccccc}
\hline System & Buffer & HEPES & MOPSO & Bicine & CAPSO & CAPS & NaCl \\
\hline pH 7-10.5 & A (mM) & 0.0 & 7.1 & 5.3 & 14.9 & 0.7 & 12.6 \\
& B (mM) & 0.0 & 14.6 & 4.9 & 1.4 & 7.1 & 0.0 \\
pH 8-10.5 & A (mM) & 5.5 & 0.0 & 4.2 & 9.5 & 0.8 & 6.3 \\
& B (mM) & 0.0 & 0.0 & 10.5 & 2.5 & 7.0 & 0.0 \\
\hline
\end{tabular}


elution gradient for both buffer systems was set to $0.07 \mathrm{pH} /$ min. The chromatograms were evaluated with ChemStation software. As a reference, the same adalimumab standard as stated above was used. Statistical analysis was performed with SigmaPlot 13.0 software.

\section{Results and discussion}

\section{Assessment of the method's appropriateness concerning the cell culture matrix}

Since the IEX method has already been thoroughly verified, this study is entirely focused on the adaptation for cell culture samples. Critical considerations were taken in terms of possible matrix effects and the qualitative evaluation of the resulting peak areas.

a
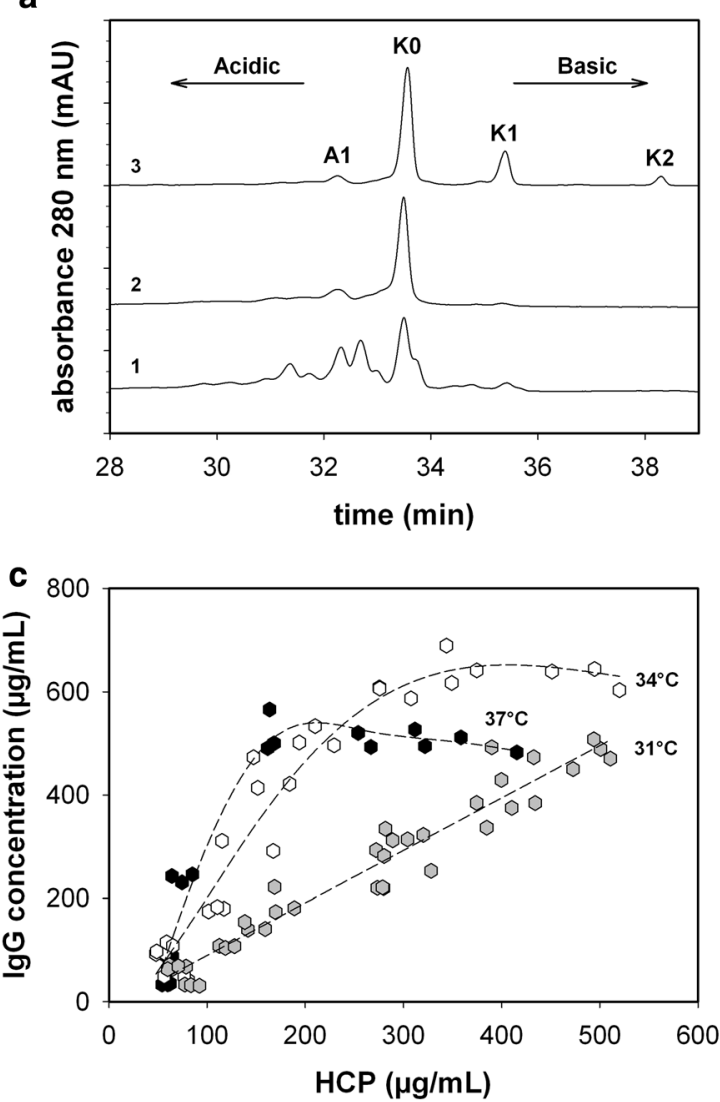

Fig. 1 a HPLC-chromatogram of a recombinantly produced protein measured directly from the supernatant at harvest (1), the same sample but pre-purified via Protein A (2) and the adalimumab reference (3), using the $\mathrm{pH} 7$ buffer system. b Amount of IgG as a function of the integrated total peak area for the shaker data [grey-filled circle] and control standards [filled triangle]. c Amount of host cell proteins (HCPs) and IgG of fed-batch experiments at $37{ }^{\circ} \mathrm{C}$ [filled hexagon],
The recombinantly produced anti-TNF-alpha antibody was compared to a pharmaceutical adalimumab reference, which is a well-described $\mathrm{mAb}$ [21]. The reference standard analyzed by a CEX with a linear $\mathrm{pH}$ gradient exhibited a distinct peak distribution (see Fig. 1a). Due to the use of a strictly $\mathrm{pH}$-dependent system, the charge variants were only separated according to their net surface charge, where acidic forms were eluted prior to the neutral and basic variants. Both buffer systems (pH 7-10.5 and $\mathrm{pH} 8-10.5)$ and different flow rates were tested in terms of their applicability for supernatants. The $\mathrm{pH}$ gradient itself was set to a constant slope of $0.07 \mathrm{pH} / \mathrm{min}$. In conclusion, the $\mathrm{pH} 7$ variant resulted in a better separation of the acidic species. It is obvious that the close proximity of the calculated $\mathrm{pI}$ of the $\mathrm{mAb}(8.60)$ and the starting $\mathrm{pH}$ conditions affect the separation profile of the acidic isoforms. The tested flow rates, between 0.5 and $1 \mathrm{~mL} / \mathrm{min}$, did not significantly affect the measurement's resolution characteristics. This confirms the
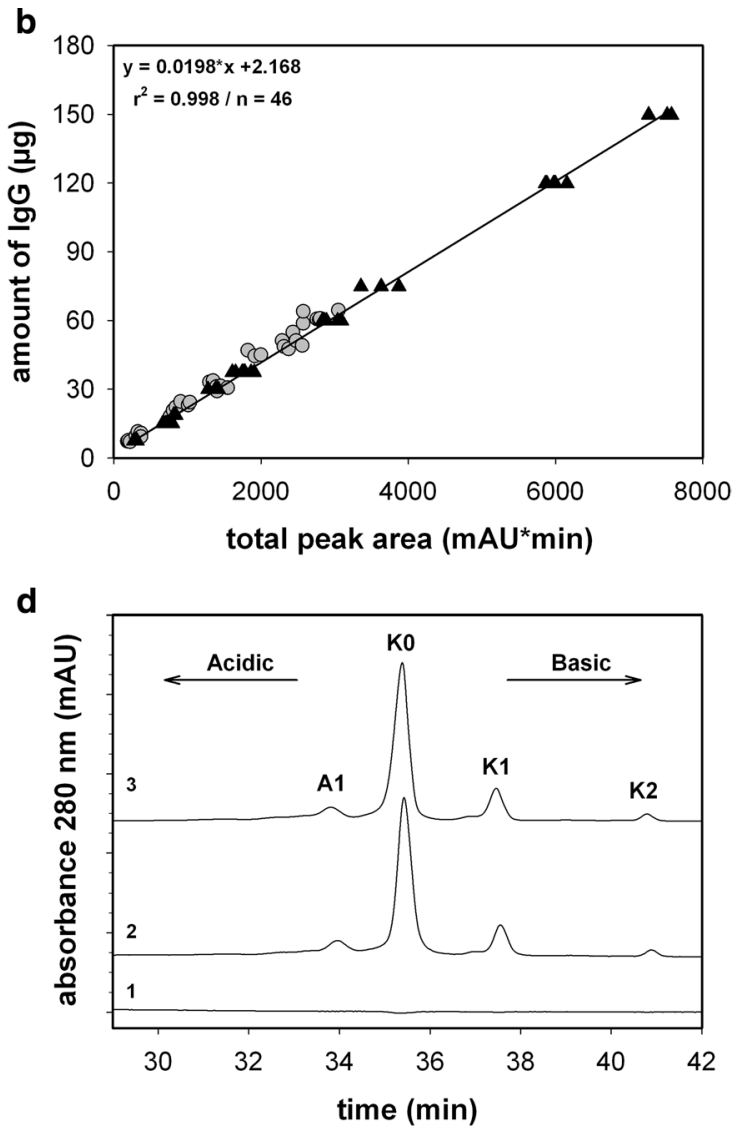

$34{ }^{\circ} \mathrm{C}$ [unfilled hexagon] and $31{ }^{\circ} \mathrm{C}$ [grey hexagon]. d CEX chromatogram of a mocking supernatant (1), a standard spiked into the same supernatant (2) and the standard (3). KO main variant, K1 1-lysine variant, $K 2$ 2-lysine variant, $A 1$ acidic variant without further characterization. Linear regression was performed on the adalimumab dataset 
excellent mass transfer properties of the core shell particlebased stationary phase. Therefore, the $\mathrm{pH} 7$ buffer system was chosen and the flow rate was set to $1 \mathrm{~mL} / \mathrm{min}$ for the following studies. Due to the fact that the resolution was already sufficient, no attention was paid to optimize the running conditions any further.

Under these conditions, several samples and the reference standard in different matrices were analyzed as shown in Fig. 1. The most abundant variant was allocated to the main variant and marked as $\mathrm{K} 0$, where $\mathrm{K} 0$ represents the complete cleavage of the C-terminal lysine. In the basic region, two peaks were evident, which caused incomplete lysine clipping, enumerated as $\mathrm{K} 1$ and $\mathrm{K} 2$, where one or both lysine residues remained attached to the heavy chain C-termini [21]. In the acidic area, there was only one pronounced peak (A1) evident for the standard. The anti-TNFalpha antibody produced in our process revealed a different chromatographic pattern. The main variant (K0) was identified at a similar retention time, while the basic and the acidic region exhibited more variants. These differences are not remarkable due to the fact that the reference standard was a purified API, while the anti-TNF-alpha antibody expressed in a certain cell line with a defined media was produced under variable process conditions and not purified at all.

During repeated test sequences, it became apparent that slight variabilities in the buffer system resulted in minor retention time shifts caused by these sensitive gradients (see Fig. 1a, d). Consequently, the reference material was analyzed each time when a new buffer was prepared, both to control the performance characteristic and to ensure correct integration. To make the set of data comparable, the retention time was normalized. This was done by dividing the retention time $t_{i}$ by the retention time of the main variant $t_{\text {main }}$ peak. Thereafter, peak area integration was performed within a $t_{\text {normalized }}$ of $0.76-1.23$ for all analyses; in the following, this is referred to as the total peak area.

After the performance optimization, the reference standard and various samples were evaluated according different criteria. For the indication, whether or not the samples were affected by matrix proteins, both supernatant samples $(n=36)$ and standards $(n=46)$ were analyzed. The relationship between the total area and the amount of IgG, previously determined with BLI was assessed. The linear relationship of both indicated that the established performance was sufficient to obtain reliable data for standards and supernatants (see Fig. 1b).

Furthermore, for a comprehensive quantitative evaluation, it is important that the interprecision of the distribution is appropriate. The calculated coefficient of variation $(\mathrm{CV})$ for the acidic, main and basic variants of the control standard $(n=15)$ was below $10 \%$ for all variants (see Table 2). These results are in accordance with the comprehensively verified data published by Lingg et al.
Table 2 Charge heterogeneity distribution consistency of the control standards and supernatant samples determined by the described CEX method using the $\mathrm{pH} 7$ buffer system

\begin{tabular}{lll}
\hline Sample & Variant & Relative area $\pm \sigma(\%)$ \\
\hline $\begin{array}{c}\text { Pharmaceutical standard } \\
(n=15)\end{array}$ & Acidic & $16.2 \pm 1.3$ \\
& Main & $59.2 \pm 1.6$ \\
Fed-batch samples drawn & Basic & $24.5 \pm 0.8$ \\
at day 4 $(n=8)$ & Main & $69.2 \pm 3.0$ \\
& Basic & $24.6 \pm 2.5$ \\
\hline
\end{tabular}

[25]. For demonstrating the charge distribution reproducibility of the recombinantly produced mAbs in supernatants, as fed-batch samples, which were collected at day 4 , were analyzed. Day 4 was at the end of the batch phase assuming that the experiments were performed in a similar manner. All temperature shifts were performed after the batch phase. The acidic and main variant distribution exhibited a CV of $4 \%$ and $10 \%$, respectively $(n=8)$. The $\mathrm{CV}$ of the basic variant area was higher at $26 \%$, caused by the low amount of this variant (see Table 2). Compared to the standard, the quantitative distribution of the fed-batch sample at day 4 was different. Acidic species were the most abundant variants, evidencing a possible impact of the chosen bioprocess conditions.

Although it can be shown that a linear relationship between the total area and the amount of $\operatorname{IgG}$ exists, the variable amount of HCPs could falsify the chromatographic results, for instance, in the later stages of the cell culture process, when the viability decreases and the cells start to lyse. The HCP population is highly heterogeneously composed, but the majority should exhibit a pI below 7 and a molecular weight lower than $150 \mathrm{kDa}[39,40]$. The ratio of HCP to IgG titer ranged between 20 and $50 \%$ and was independent of the feeding strategy (see Fig. 1c). Co-elution of any proteins or antibody fragments was not observed. Silver-stained fractionated samples revealed only one pronounced band at around $150 \mathrm{kDa}$ (data not shown). Additionally, in the mock control, where the HCP content was even higher (up to $700 \mu \mathrm{g} / \mathrm{mL}$ ), no additional peak occurred within the elution period of the mAb. In this respect, only a slight baseline drift of around 0.2-0.4 milli-absorbance units was detectable, primarily indicating an effect of supernatant compositions, which is not caused by HCPs. Spiking experiments in the mock control supernatant confirmed the assumption that HCPs do not affect the elution pattern (see Fig. 1d). Therefore, the contribution of HCPs and the culture supernatant matrix to the measured charge variant distribution was considered as not significant. Co-elution of any other proteins or other cell culture components did not affect the quality of the obtained data. 
In summary, the results indicate that the determination of $\mathrm{mAb}$ charge distribution directly from supernatants is practicable. It has also been shown that the method is reliable and reproducible.

\section{Process monitoring of cell culture processes}

The optimized method was used to monitor mAb charge heterogeneity during a cell culture process to elucidate the influences of temperature and glucose concentration.

The batch phase exhibited similar trends in all measured variables for each experiment. The process temperatures had a significant impact on overall productivity, growth rates and viability of the cells (see Fig. 2). Such observations have already been described in several publications [41-44]. Reduced process temperature can, moreover, be used for the proliferation control of cell culture processes [45]. Even though glucose concentrations in the cultures varied from 2 to $15 \mathrm{~g} / \mathrm{L}$ (Fig. 2d), depending on the feed and temperature, almost no impact on the monitored process variables could be detected. Only the osmolality could be partly linked to the glucose concentration in the supernatant, which ranged from 345 (high glucose) to $233 \mathrm{mOsm} / \mathrm{kg}$ (low glucose) (see Fig. 2d, e). Lactate production was only observed in the batch phase, while consumption took place during the remaining process (Fig. 2c), independent of the temperature and glucose profile. Interestingly, no difference in specific nutrient uptake and byproduct formation rates was determined, due neither to the elevated glucose level nor to the change in process temperature. $q_{\text {gluc }}$ was independent in respect of the feed or process temperature used (see Fig. 2f), with such behavior previously reported in other publications [46, 47]. The observation may be correlated with the fact that glucose uptake is not only dependent on the amount of glucose but also on amino acids such as leucine, lysine and serine [48].

To evaluate the impact of process variation on charge distribution, several fed-batch samples from day 4, followed by samples after the temperature shift until the harvest criteria with a viability of $70 \%$, were applied to the CEX column $(n=36)$. In turn, it became obvious that variation in the process parameters, glucose concentration and temperature affected the charge variant distributions to a great extent (see Fig. 3). Since the glucose concentration had no apparent influence on cell metabolism, it was supposed to have affected the $\mathrm{mAb}$ charge distribution in an extracellular manner (see Fig. 3a, c, e, g). This is also evidenced by the fact that the $\mathrm{K} 0$ main proportion correlates linearly with the percentage of acidic species (Fig. 3a). The resulting basic species are in opposition to this observation due to the fact that they mostly derive from incomplete C-terminal lysine processing, which is a known intracellular process (see Fig. 3b, d, f).
Under these defined process conditions, the basic species were generally rare, which suggests that C-terminal lysine processing occurred almost completely. However, process temperature predominantly affected the basic variant formation. As the amount of basic species was independent of the amount of the main variant, no trend could be identified; only two cluster regions were obvious (Fig. 3b). However, three linear relationships between the total basic area and $\mathrm{K} 0$ peak area could be determined by separating the data into three distinct groups according to the applied temperature (Fig. 3d). Evidently, lowering the temperature resulted in imperfect $\mathrm{C}$-terminal lysine processing. This temperature-based occurrence was in accordance with previously published data [49]. The expression levels and the specific activity of the enzyme carboxypeptidase (B and $\mathrm{H}$ ), which is considered to play a major role in C-terminal lysine cleavage, is temperature dependent [50,51]. Hence, the processing of C-terminal lysine clipping, an important quality attribute [16], can be influenced by process temperature and sufficiently monitored with this method. The extracellular glucose concentration had no impact at all on basic species variations (Fig. 3f). The dataset could only be divided into two distinct groups: a $37{ }^{\circ} \mathrm{C}+34{ }^{\circ} \mathrm{C}$ and a $31^{\circ} \mathrm{C}$ cluster. No correlation was evident. Thus, in conclusion, the accumulated amount of basic species was the result of an intracellular process and most probably regulated by the amount and activity of the carboxypeptidase.

The vast impact of glucose on the micro-heterogeneity of the mAb was evident. The highest main variant (K0) content was observed at $34{ }^{\circ} \mathrm{C}$ with Feed 1 (low glucose). At an elevated glucose concentration, the amount of $\mathrm{K} 0$ was significantly reduced. For instance, the process at $31{ }^{\circ} \mathrm{C}$ with Feed 3 (high glucose) resulted in the highest charge heterogeneity. Acidic variants were the most abundant variants and ranged from 60 to $90 \%$ of the total peak area. It was obvious that an increase in the acidic species was attended by a decrease in the main variant (see Fig. 3a). The process at $31{ }^{\circ} \mathrm{C}$, however, exhibited a slight parallel shifted linear correlation, due to the increased amount of basic species. At $31{ }^{\circ} \mathrm{C}$, the proportion of basic variants was, on average, $5 \%$ higher than in the case of the other processes, which resulted in a decreased offset value of around the same proportion. However, both correlations exhibited a similar slope (Fig. 3a). The acidic heterogeneity was mainly dependent on the feed used; thus, an increase in glucose in the supernatant resulted in an enriched fraction of acidic variants (see Fig. 3c, e). It was lowest at $34{ }^{\circ} \mathrm{C}$ and $37{ }^{\circ} \mathrm{C}$ when a low glucose feed was applied. An induction of $\mathrm{mAb}$ alteration effects, due to osmolality and differences in the elution pattern, as reported by Schmelzer and Miller [52], was not evident. Acidic variant formation was mainly provoked by two parameters: the increasing total amount of main variants and incremental glucose concentration in the supernatant. Interestingly, 

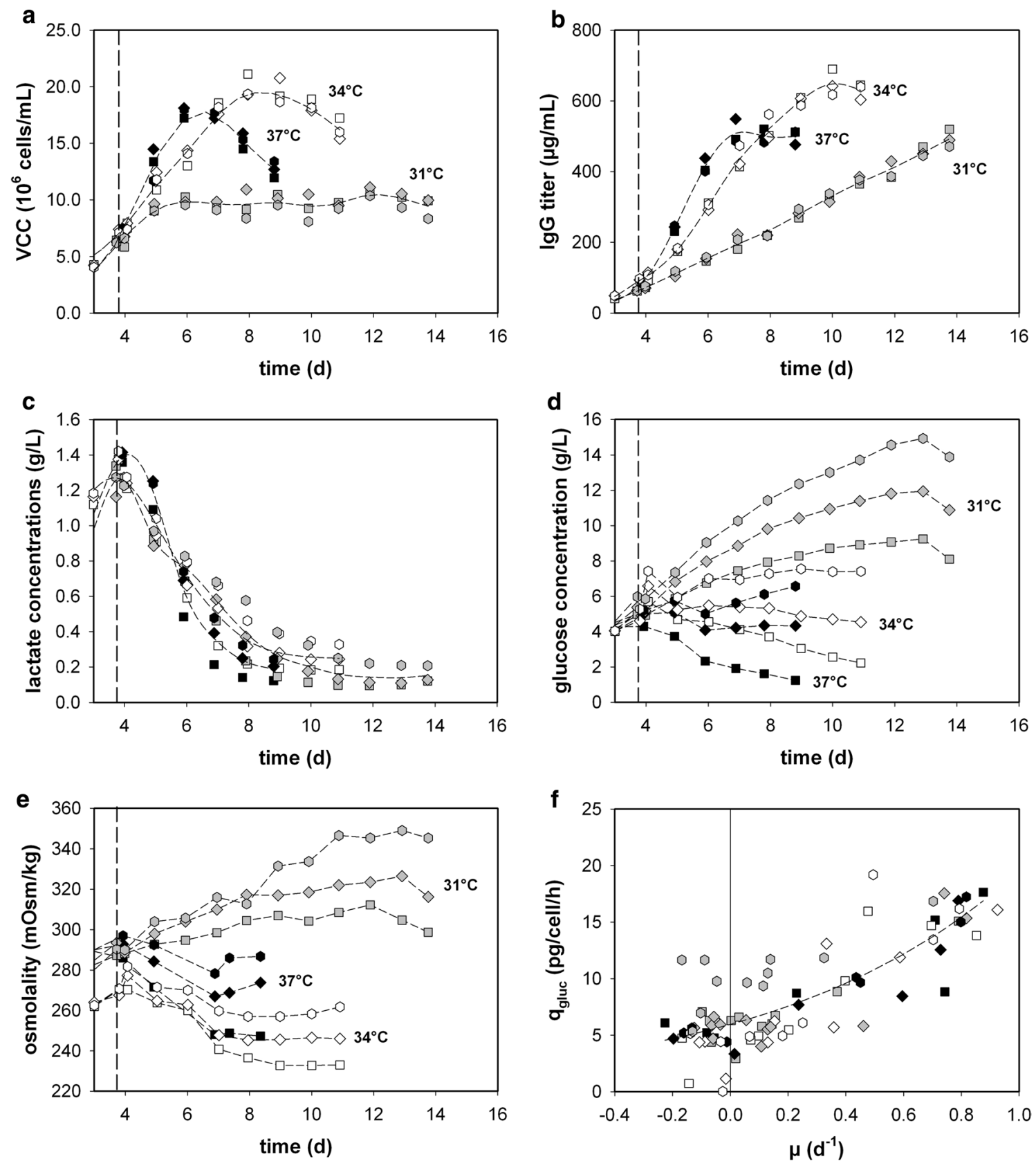

Fig. 2 Fed-batch process parameters: a VCC, b protein titer, c lactate and $\mathbf{d}$ glucose concentration and e osmolality as a function of process time. $\mathbf{f}$ Specific glucose consumption $\left(q_{\text {gluc }}\right)$ as a function of the growth rate $(\mu) .37{ }^{\circ} \mathrm{C}+$ Feed 1 [filled square], $37{ }^{\circ} \mathrm{C}+$ Feed 2 [filled diamond], $37{ }^{\circ} \mathrm{C}+$ Feed 3 [filled hexagon], $34{ }^{\circ} \mathrm{C}+$ Feed 1 [unfilled

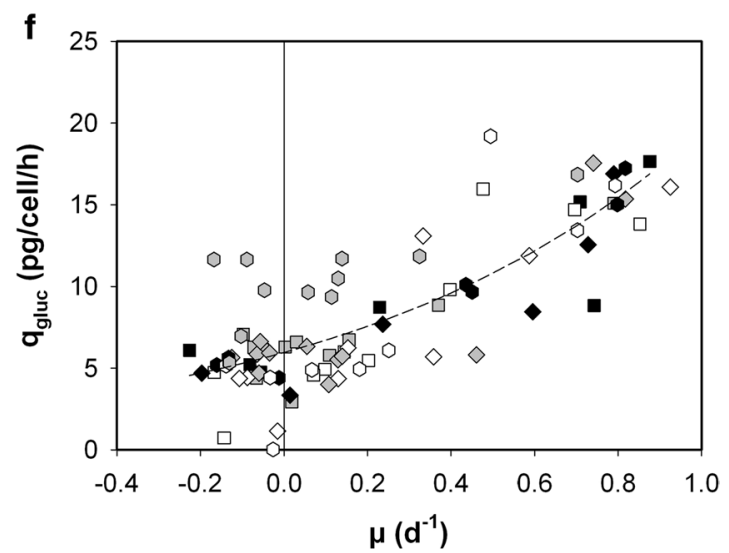

square], $34{ }^{\circ} \mathrm{C}+$ Feed 2 [unfilled diamond], $34{ }^{\circ} \mathrm{C}+$ Feed 3 [unfilled hexagon], $31{ }^{\circ} \mathrm{C}+$ Feed 1 [grey square], $31{ }^{\circ} \mathrm{C}+$ Feed 2 [grey diamond], $31{ }^{\circ} \mathrm{C}+$ Feed 3 [grey hexagon]. Fed-batch process started at day 3 . Vertical dashed line indicates temperature shift $(\approx$ day 4$)$. Short dashed lines indicate trends

regarding the latter, a saturation plateau was reached. At glucose concentrations higher than $7 \mathrm{~g} / \mathrm{L}$, the ratio of the total acidic area to the $\mathrm{K} 0$ main peak area remained almost unaffected (Fig. 3e).

According to these results, three assumptions were made: first, during those bioprocesses, acidic variants mainly evolved from the main ones already present in the supernatant; second, considering that the different feeds

had no influence on any major process parameter, among others, biomass and productivity, the glucose concentration in the supernatant directly influenced the formation of acidic variants; and, third, there was a predefined number of possible glucose-inducible acidic variants. Under the selected process conditions, glycation, the non-enzymatic attachment of a reactive glucose to a protein, was most likely a feasible cause of acidic peak formation. It has 
a

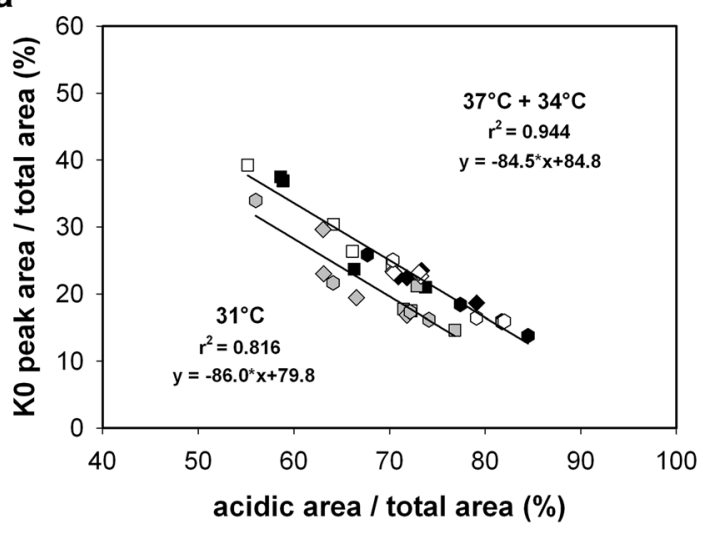

C

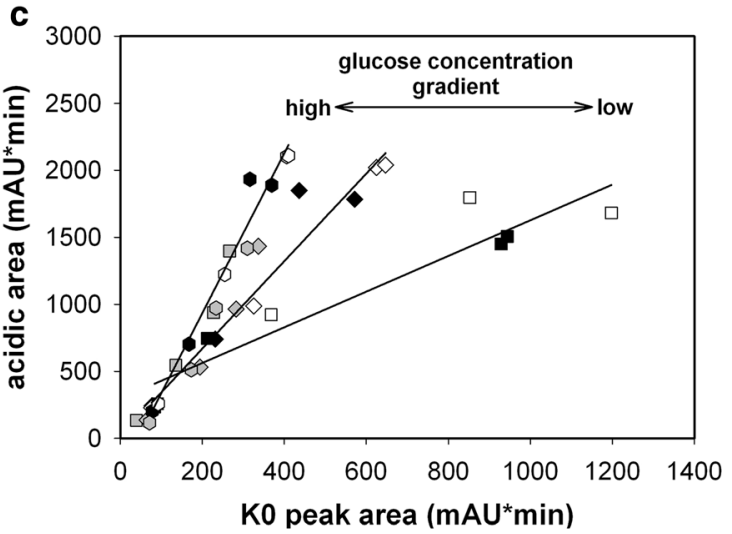

e
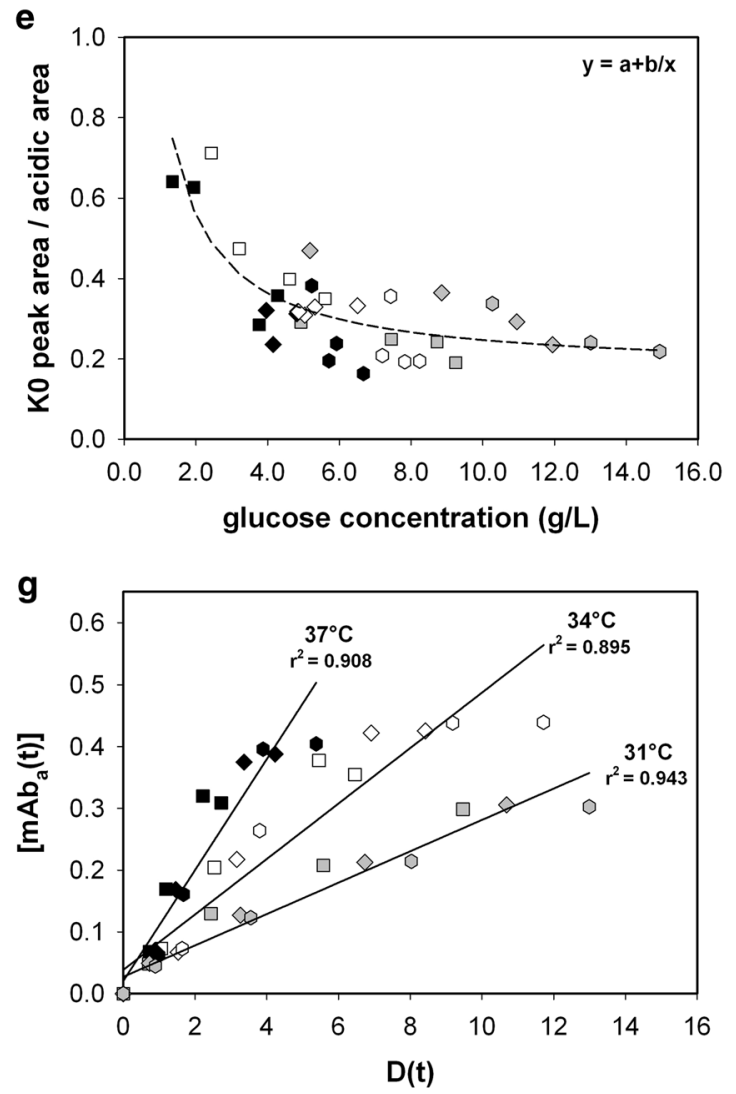

b

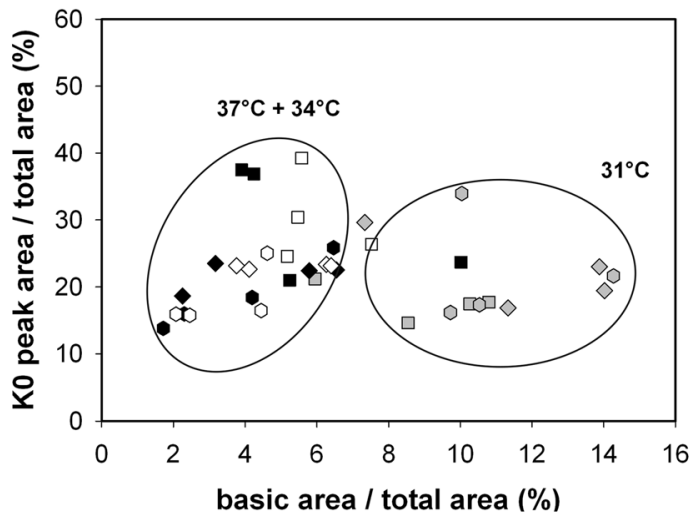

d
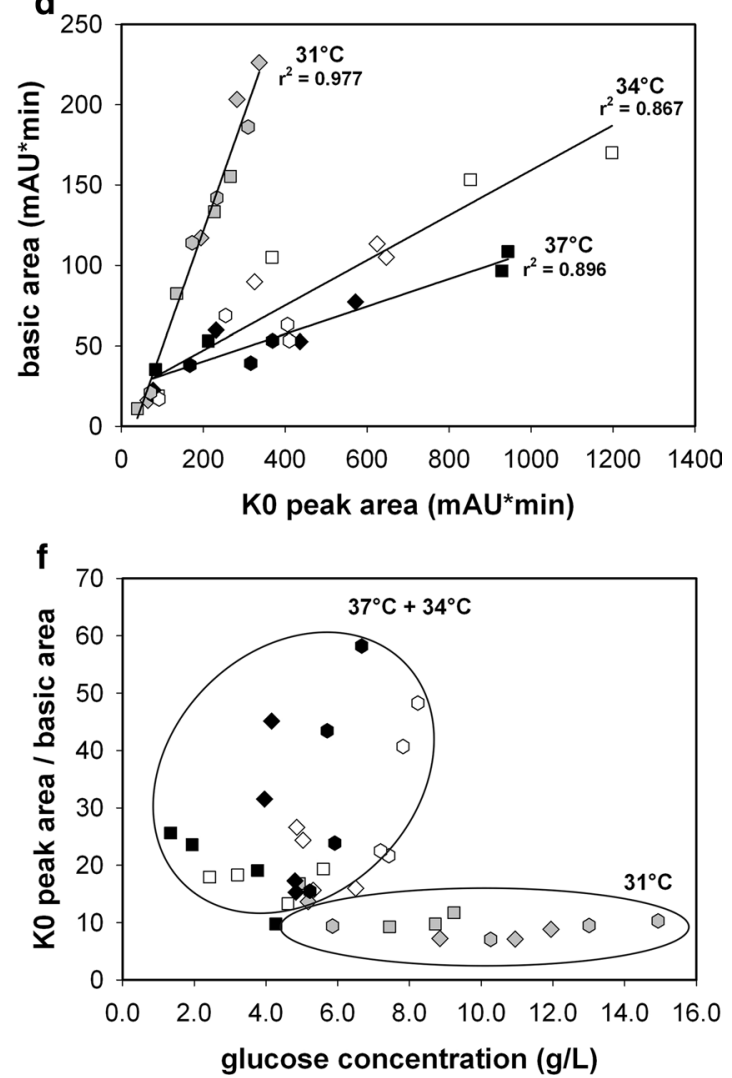
4 Fig. 3 K0 main peak ratio as a function of the acidic (a) and basic variant (b) ratio. $\mathbf{c}$ Acidic area and $\mathbf{d}$ basic area as a function of the $\mathrm{K} 0$ main peak area. The ratio of the K0 peak area to the e acidic area and the $\mathbf{f}$ basic area as a function of glucose concentration. $\mathbf{g}$ Acidic peak area concentration at time point $t$ as a function of $D(t)$, that is, the product of newly built $\mathrm{IgG}$ concentration and glucose concentration integrated over time. $37{ }^{\circ} \mathrm{C}+$ Feed 1 [filled square], $37{ }^{\circ} \mathrm{C}+\mathrm{Feed}$ 2 [filled diamond], $37{ }^{\circ} \mathrm{C}+$ Feed 3 [filled hexagon], $34{ }^{\circ} \mathrm{C}+$ Feed 1 [unfilled square], $34{ }^{\circ} \mathrm{C}+$ Feed 2 [unfilled diamond], $34{ }^{\circ} \mathrm{C}+$ Feed 3 [unfilled hexagon], $31{ }^{\circ} \mathrm{C}+$ Feed 1 [grey square], $31{ }^{\circ} \mathrm{C}+\mathrm{Feed}$ 2 [grey diamond], $31^{\circ} \mathrm{C}+$ Feed 3 [grey hexagon]

already been related to the protein content and the glucose concentration [12] and also seems to be substantiated by this study.

Due to that fact that acidic species, induced by glucose, were most likely extracellularly formed, a second-order reaction, as proposed for typical non-reversible glycation reaction [53], was constructed. Unlike Yuk et al. [53], we set up some constraints. First, only a certain amount of the antibody is susceptible to be transformed into an acidic species. Second, if one antibody is not transformed within the certain time period, then a transformation will not occur during the rest of the process. We defined the second-order reactions as follow (Eqs. 1-3):

$[\Delta \mathrm{mAb}]+[$ glucose $] \rightarrow\left[\mathrm{mAb}_{\text {acidic }}\right]$

Setting up the second-order reaction in its differential form, as well as integrating and rearranging, yielded:

$\left[\mathrm{mAb}_{\text {acidic }}\right](t)=k \times D(t)$

with

$D(t)=\int_{t_{0}}^{t_{i}}[\Delta \mathrm{mAb}] \times[$ glucose $] \times \mathrm{d} t$,

where $\left[\mathrm{mAb}_{\text {acidic }}\right](t)$ represents the concentration of the acidic variants at time point $t_{i}, k$ is the reaction constant and $D(t)$ is the integrated product at time point $t_{i}$ of the newly built IgG and the glucose concentration. If the second-order reaction assumption is true, the plotting of $\left[\mathrm{mAb}_{\text {acidic }}\right](t)$ against $D(t)$ should yield in a straight line with a slope of $k$ (Fig. 3g).

Evidently, the data are separated into three distinct groups, in respect of their applied process temperature. A linear regression was carried out. In conclusion, the reaction rate, and consequently the slopes of the representative lines, were dependent on the process temperature: 0.09 , 0.04 and $0.03 \frac{g_{\text {acidic }} L}{g_{\operatorname{lgG}} g_{\text {glucose }} d}$, for $37^{\circ} \mathrm{C}, 34^{\circ} \mathrm{C}$ and $31^{\circ} \mathrm{C}$, respectively. These findings are in agreement with published research data. Typical antibody alteration effects, which result in acidic variant formation, for instance, deamidation $[54,55]$ or glycation $[56,57]$, can be modulated by temperature [51].
Finally, it can be speculated that the glycosylation pattern may also contribute to the acidic charge heterogeneity (Fig. 4a). Nevertheless, this is not the case, because anti-TNF-alpha antibodies do not contain any sialic acids, while the proportion of high mannose types is similar to the reference material [13, 21]. Additionally, the BAC analysis indicated that a substantial proportion of the $\mathrm{mAb}$ was glycated (Fig. 4b). The reference material, when incubated in a high glucose solution, exhibited a peak at a similar retention time. Thus, in conclusion, it was determined that glycation was the main driver for the formation of variable amounts of acidic species under the adjusted process conditions.

To the best of our knowledge, this is the first study that provides insights into the formation of charge variants during a cell culture process without using any pre-purification steps. Evidently, within the chosen process conditions, the formation of variable acidic species is of dominant importance. To achieve an understanding of consistent product quality, appreciating the mechanism of charge variant formation is inevitable. Rapid determination of the charge distribution pattern can significantly facilitate process optimization. This can be useful for process development and control of antibody and biosimilar production. Medium composition effects, such as glucose concentration and physical variations (e.g., temperature), on the generation of charge variants can be analyzed accordingly; thus, product quality attributes can be determined very early on in the process chain. That said, when assigning the occurring variants to distinct posttranslational modifications, a more detailed and thorough characterization is necessary. The chromatographic pattern alone does not imply the occurred modifications [58]. Combination with other methods, such as LC-MS or BAC, could help to significantly improve the understanding of the mechanism of peak formation.

\section{Conclusion}

In this work, we established a process-monitoring tool for the determination of charge heterogeneity of mAbs directly from cell culture supernatants. This method is based on cation exchange chromatography using a linear, basic $\mathrm{pH}$ gradient, with which cell culture supernatant samples can be directly analyzed for $\mathrm{mAb}$ charge heterogeneity without the need for prior protein purification. This represents a potential powerful tool for process development, since changes in product quality due to changes in process parameters can be detected earlier. It also has potential to serve as an in-process control method for any mAb production process, which can offer invaluable process knowledge. Under the Quality by Design paradigm, this increase in knowledge about the relationship 


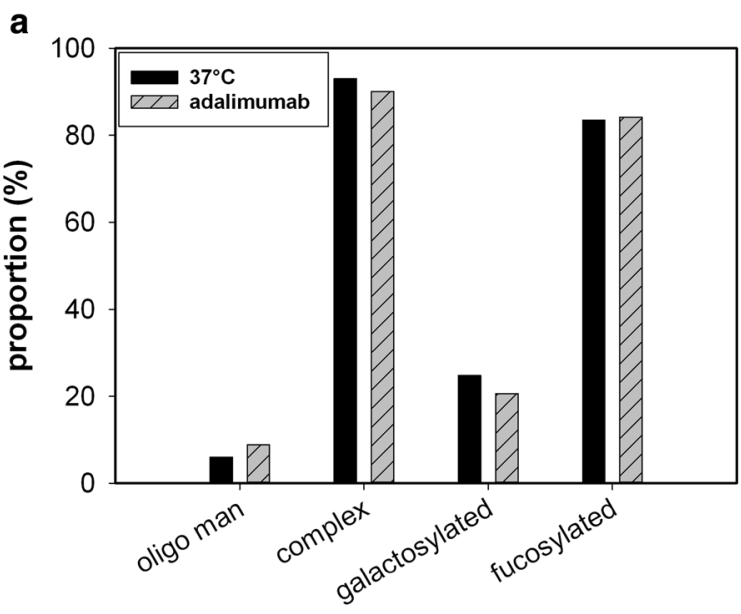

Fig. 4 a Type of detected glycoforms for reference, for the recombinant produced $\mathrm{mAb}$ at $37^{\circ} \mathrm{C}$. b BAC chromatogram of the reference material (1), the reference incubated in $0.19 \mathrm{M}$ glucose solution for

of product and quality parameters can lead to a more consistent product quality through improved process control.

We also present a case study, in which the process parameter glucose level and temperature where analyzed for their effect on product quality. We showed that target protein production was predominantly affected by the process temperature, whereas the acidic variant formation and thus the product quality were highly influenced by the glucose level in the cell environment. The formation of acidic variants could be conclusively linked to an extracellular mechanism. These observations confirm the importance of the control of glucose level to ensure consistent high-quality mAb output. The process temperature, however, remains important as well, since, next to the titer, it secondarily affected the rate of acidic peak formation as well as basic variant formation.

However, charge heterogeneity of a mAb can be an adequate fingerprinting technique to confirm the desired product quality attributes, already early in the process chain. Depending on the mAb, the method can be adapted to gain information in an even shorter period of time. In general, this method will add great value to process optimization.

Acknowledgements Open access funding provided by University of Natural Resources and Life Sciences Vienna (BOKU). We would like to thank Florian Strobl for the idea to perform this study, as well as Clemens Keinprecht, Katarina Kljajic and Gabi Lhota, along with the whole PAT-Plant project team, for their practical contribution to this work. The project was funded by the Austrian Research Promotion Agency (FFG) and Bilfinger Industrietechnik Salzburg (Competence Headquarters, 849725).

\section{Compliance with ethical standards}

Conflict of interest The authors declare no commercial or financial conflicts of interest.

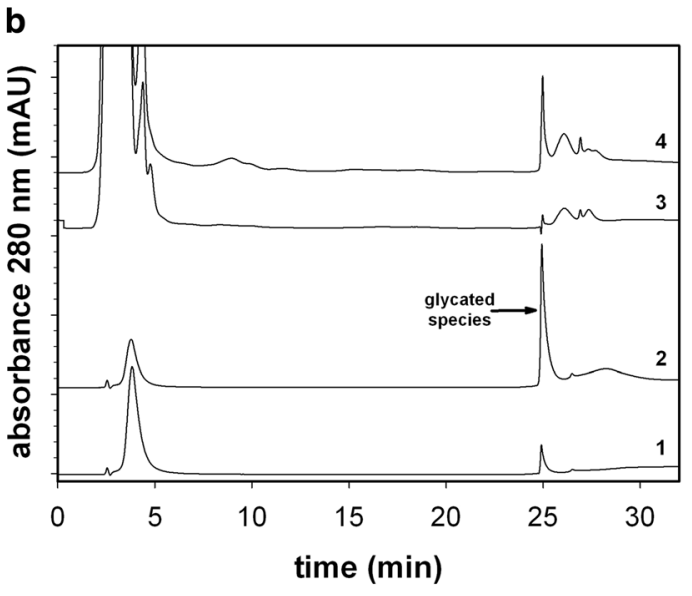

$68 \mathrm{~h}$ at $37^{\circ} \mathrm{C}(2)$, mocking supernatant from the host cell line (3), and a supernatant sample of a fed-batch process with a high number of acidic variants $(\approx 90 \%)(4)$

Open Access This article is distributed under the terms of the Creative Commons Attribution 4.0 International License (http://creativeco mmons.org/licenses/by/4.0/), which permits unrestricted use, distribution, and reproduction in any medium, provided you give appropriate credit to the original author(s) and the source, provide a link to the Creative Commons license, and indicate if changes were made.

\section{References}

1. Ecker DM, Jones SD, Levine HL (2015) The therapeutic monoclonal antibody market. MAbs 7(1):9-14

2. Udpa N, Million RP (2016) Monoclonal antibody biosimilars. Nat Rev Drug Discov 15(1):13-14

3. Kunert R, Reinhart D (2016) Advances in recombinant antibody manufacturing. Appl Microbiol Biotechnol 100(8):3451-3461

4. Rathore $\mathrm{S}$ et al (2015) Fermentanomics: relating quality attributes of a monoclonal antibody to cell culture process variables and raw materials using multivariate data analysis. Biotechnol Prog 31(6): 1586-1599

5. Beyer M, Schuster A Jungbauer, Lingg N (2018) Microheterogeneity of recombinant antibodies: analytics and functional impact. Biotechnol J 13(1):1-26

6. Goncalves J, Araujo F, Cutolo M, Fonseca E (2016) Biosimilar monoclonal antibodies: preclinical and clinical development aspects. Clin Exp Rheumatol 34(4):698-705

7. Sha S, Agarabi C, Brorson K, Lee DY, Yoon S (2016) N-glycosylation design and control of therapeutic monoclonal antibodies. Trends Biotechnol 34(10):835-846

8. Nidhi K, Indrajeet S, Khushboo M, Gauri K, Sen DJ (2011) Hydrotropy: a promising tool for solubility enhancement: a review. Int J Drug Dev Res 3(2):26-33

9. Liu et al (2014) In vitro and in vivo modifications of recombinant and human IgG antibodies. MAbs 6(5):1145-1154

10. Singh SK, Narula G, Rathore AS (2016) Should charge variants of monoclonal antibody therapeutics be considered critical quality attributes? Electrophoresis 37(17-18):2338-2346

11. Jefferis R (2016) Posttranslational modifications and the immunogenicity of biotherapeutics. J Immunol Res 2016:1-15

12. Quan C et al (2008) A study in glycation of a therapeutic recombinant humanized monoclonal antibody: where it is, how it got 
there, and how it affects charge-based behavior. Anal Biochem 373(2):179-191

13. Du Y, Walsh A, Ehrick R, Xu W, May K, Liu H (2012) Chromatographic analysis of the acidic and basic species of recombinant monoclonal antibodies. MAbs 4(5):578-585

14. Hintersteiner B, Lingg N, Janzek E, Mutschlechner O, Loibner H, Jungbauer A (2016) Microheterogeneity of therapeutic monoclonal antibodies is governed by changes in the surface charge of the protein. Biotechnol J 11(12):1617-1627

15. Hintersteiner B et al (2016) Charge heterogeneity: basic antibody charge variants with increased binding to Fc receptors. MAbs $8(8): 1548-1560$

16. van den Bremer ETJ et al (2015) Human IgG is produced in a pro-form that requires clipping of C-terminal lysines for maximal complement activation. MAbs 7(4):672-680

17. Fekete S, Gassner AL, Rudaz S, Schappler J, Guillarme D (2013) Analytical strategies for the characterization of therapeutic monoclonal antibodies. Trends Anal Chem 42:74-83

18. Wang F, Peklansky B, Anderson C, Wang Y, Rustandi RR (2012) Improved ion-exchanged HPLC method in mab using ph gradient and its comparison with cIEF. J Liq Chromatogr Relat Technol 35(9):1259-1269

19. Fekete S, Beck A, Veuthey JL, Guillarme D (2015) Ion-exchange chromatography for the characterization of biopharmaceuticals. J Pharm Biomed Anal 113:43-55

20. Joshi V, Kumar V, Rathore AS (2015) Rapid analysis of charge variants of monoclonal antibodies using non-linear salt gradient in cation-exchange high performance liquid chromatography. $\mathrm{J}$ Chromatogr A 1406:175-185

21. Tebbey PW, Varga A, Naill M, Clewell J, Venema J (2015) Consistency of quality attributes for the glycosylated monoclonal antibody Humira ${ }^{\circledR}$ (adalimumab). MAbs 7(5):805-811

22. Wagner-Rousset E et al (2017) Development of a fast workflow to screen the charge variants of therapeutic antibodies. J Chromatogr A $1498: 147-154$

23. Farnan D, Moreno GT (2009) Multiproduct high-resolution monoclonal antibody charge variant separations by $\mathrm{pH}$ gradient ionexchange chromatography. Anal Chem 81(21):8846-8857

24. Lingg N, Tan E, Hintersteiner B, Bardor M, Jungbauer A (2013) Highly linear $\mathrm{pH}$ gradients for analyzing monoclonal antibody charge heterogeneity in the alkaline range. J Chromatogr A 1319:65-71

25. Lingg N, Berndtsson M, Hintersteiner B, Schuster M, Bardor M, Jungbauer A (2014) Highly linear pH gradients for analyzing monoclonal antibody charge heterogeneity in the alkaline range: validation of the method parameters. J Chromatogr A 1373:124-130

26. Michels DA, Tu AW, McElroy W, Voehringer D, Salas-Solano O (2012) Charge heterogeneity of monoclonal antibodies by multiplexed imaged capillary isoelectric focusing immunoassay with chemiluminescence detection. Anal Chem 84(12):5380-5386

27. Van Beers MMC, Bardor M (2012) Minimizing immunogenicity of biopharmaceuticals by controlling critical quality attributes of proteins. Biotechnol J 7(12):1473-1484

28. Sommeregger W, Sissolak B, Kandra K, von Stosch M, Mayer M, Striedner G (2017) Quality by control: towards model predictive control of mammalian cell culture bioprocesses. Biotechnol J 12(7):1-7

29. Cohn EJ et al (1946) Preparation and properties of serum and plasma proteins. IV. A system for the separation into fractions of the protein and lipoprotein components of biological tissues and fluids. J Am Chem Soc 68(3):459-475

30. Bergmann-Leitner ES, Mease RM, Duncan EH, Khan F, Waitumbi J, Angov E (2008) Evaluation of immunoglobulin purification methods and their impact on quality and yield of antigen-specific antibodies. Malar J 7(1):129
31. Gagnon P, Nian R (2016) Conformational plasticity of IgG during protein A affinity chromatography. J Chromatogr A 1433:98-105

32. Zboray $\mathrm{K}$ et al (2015) Heterologous protein production using euchromatin-containing expression vectors in mammalian cells. Nucleic Acids Res 43(16):e102

33. Strober W (2001) Trypan blue exclusion test of cell viability. Curr Protoc Immunol Appendix 3B:1-2

34. Reinhart D, Damjanovic L, Kaisermayer C, Kunert R (2015) Benchmarking of commercially available $\mathrm{CHO}$ cell culture media for antibody production. Appl Microbiol Biotechnol 99(11):4645-4657

35. Zhang B, Mathewson S, Chen H (2009) Two-dimensional liquid chromatographic methods to examine phenylboronate interactions with recombinant antibodies. J Chromatogr A 1216:5676-5686

36. Gruber C, Altmann F (2015) Site-specific glycosylation profiling using liquid chromatography-tandem mass spectrometry (LCMS). In: Castilho A (ed) Glyco-engineering. Humana Press, New York, pp 407-415

37. Loos C, Gruber F, Altmann U, Mehofer F, Hensel Grandits M (2014) Expression and glycoengineering of functionally active heteromultimeric IgM in plants. PNAS 111(17):6263-6268

38. Stadlmann J, Pabst M, Kolarich D, Kunert R, Altmann F (2008) Analysis of immunoglobulin glycosylation by LC-ESI-MS of glycopeptides and oligosaccharides. Proteomics 8(14):2858-2871

39. Jin M, Szapiel N, Zhang J, Hickey J, Ghose S (2010) Profiling of host cell proteins by two-dimensional difference gel electrophoresis (2D-DIGE): implications for downstream process development. Biotechnol Bioeng 105(2):306-316

40. Tcheliessnig L, Konrath J, Bates R, Jungbauer A (2013) Host cell protein analysis in therapeutic protein bioprocessing-methods and applications. Biotechnol J 8(June):655-670

41. Moore et al (1997) Effects of temperature shift on cell cycle, apoptosis and nucleotide pools in CHO cell batch cultues. Cytotechnology 23(1-3):47-54

42. Furukawa K, Ohsuye K (1999) Enhancement of productivity of recombinant alpha-amidating enzyme by low temperature culture. Cytotechnology 31(1-2):85-94

43. Yoon SK, Song JY, Lee GM (2003) Effect of low culture temperature on specific productivity, transcription level, and heterogeneity of erythropoietin in Chinese hamster ovary cells. Biotechnol Bioeng 82(3):289-298

44. Trummer E et al (2006) Process parameter shifting: Part I. Effect of DOT, $\mathrm{pH}$, and temperature on the performance of Epo-Fc expressing $\mathrm{CHO}$ cells cultivated in controlled batch bioreactors. Biotechnol Bioeng 94(6):1033-1044

45. Kumar N, Gammell P, Clynes M (2007) Proliferation control strategies to improve productivity and survival during $\mathrm{CHO}$ based production culture: a summary of recent methods employed and the effects of proliferation control in product secreting $\mathrm{CHO}$ cell lines. Cytotechnology 53(1-3):33-46

46. Vergara M et al (2014) Differential effect of culture temperature and specific growth rate on $\mathrm{CHO}$ cell behavior in chemostat culture. PLoS One 9(4):1-6

47. Fan Y et al (2015) Amino acid and glucose metabolism in fedbatch $\mathrm{CHO}$ cell culture affects antibody production and glycosylation. Biotechnol Bioeng 112(3):521-535

48. Sellick et al (2011) Metabolite profiling of recombinant $\mathrm{CHO}$ cells: designing tailored feeding regimes that enhance recombinant antibody production. Biotechnol Bioeng 108(12):3025-3031

49. Chung $S$ et al (2018) Industrial bioprocessing perspectives on managing therapeutic protein charge variant profiles. Biotechnol Bioeng 115(7):1646-1665

50. Bradley G, Yamauchi F (1996) Ostrich (Struthio camelus) Carboxy Pmifkdbn, kinetic properties am3 of the pancreatic enzyme. Science (80-.) 28(5):521-529 
51. Zhang X et al (2015) Culture temperature modulates monoclonal antibody charge variation distribution in Chinese hamster ovary cell cultures. Biotechnol Lett 37(11):2151-2157

52. Schmelzer E, Miller WM (2002) Hyperosmotic stress and elevated $\mathrm{pCO}_{2}$ alter monoclonal antibody charge distribution and monosaccharide content. Biotechnol Prog 18(2):346-353

53. Yuk $\mathrm{H}$ et al (2011) Controlling glycation of recombinant antibody in fed-batch cell cultures. Biotechnol Bioeng 108(11):2600-2610

54. An Y, Zhang Y, Mueller HM, Shameem M, Chen X (2014) A new tool for monoclonal antibody analysis application of IdeS proteolysis in IgG domain-specific characterization. MAbs 6(4):879-893

55. Tang $\mathrm{H}$ et al (2018) Insights into the generation of monoclonal antibody acidic charge variants during Chinese hamster ovary cell cultures. Appl Microbiol Biotechnol 102(3):1203-1214

56. Lee S, Krishnanchettiar S, Lateef SS, Gupta S (2007) Analyses of the in vitro non-enzymatic glycation of peptides/proteins by matrix-assisted laser desorption/ionization mass spectrometry. Int J Mass Spectrom 260(1):67-74

57. Berning WK, Quan C, Zhang YT (2017) Glycation of antibodies: Modification, methods and potential effects on biological functions. MAbs 9(4):586-594

58. Ponniah G, Nowak C, Neill A, Liu H (2017) Characterization of charge variants of a monoclonal antibody using weak anion exchange chromatography at subunit levels. Anal Biochem 520:49-57

Publisher's Note Springer Nature remains neutral with regard to jurisdictional claims in published maps and institutional affiliations. 